\title{
Review of: "The tumor patient in the COVID-19 pandemic-an interview-based study of 30 patients undergoing systemic antiproliferative therapy"
}

\section{Fatemeh Malek}

Potential competing interests: The author(s) declared that no potential competing interests exist.

This article has undoubtedly novel aspects.

Discussing how the covid 19 affects the life of Oncology patients, It could Be better if writers classify patients into age groups and also based on their disease. 